\title{
LASER-INDUCED SHOCK WAVES IN CONDENSED MATTER: SOME TECHNIQUES AND APPLICATIONS
}

\author{
S. N. LUO ${ }^{\mathrm{a}, *}$, D. C. SWIFT ${ }^{\mathrm{a}}$, T. E. TIERNEY IV ${ }^{\mathrm{a}}$, D. L. PAISLEY ${ }^{\mathrm{a}}$, G. A. KYRALA ${ }^{\mathrm{a}}$, \\ R. P. JOHNSON ${ }^{\mathrm{a}}$, A. A. HAUER ${ }^{\mathrm{a}}$, O. TSCHAUNER ${ }^{\mathrm{b}}$ and P. D. ASIMOW \\ ${ }^{a}$ Physics Division, Los Alamos National Laboratory, Los Alamos, NM 87544, USA; \\ ${ }^{\mathrm{b}}$ High-Pressure Science and Engineering Center and Department of Physics, \\ University of Nevada, Las Vegas, NV 89154, USA; ${ }^{\mathrm{c}}$ GPS Division, \\ California Institute of Technology, Pasadena, CA 91125, USA
}

\begin{abstract}
Laser-induced shock waves in condensed matter have important applications in dynamic material studies and high pressure physics. We briefly review some techniques in laser-induced shock waves, including direct laser drive, laser-launched flyer plate, quasi-isentropic loading, point and line imaging velocity interferometry, transient X-ray diffraction, spectroscopy and shock recovery, and their applications to study of equation of state, spallation, and phase transitions.
\end{abstract}

Keywords: Laser shock waves; Velocity interferometry; Transient X-ray diffraction; Ellipsometry; Equation of state; Spallation

\section{INTRODUCTION}

Experimental investigations of material properties under extreme pressure and temperature conditions employ static [1] and dynamic [2] techniques, complementary to each other and each with their own advantages and disadvantages. The envelope of dynamic loading techniques has been expanding, ranging from conventional powder and gas gun, high explosives, pulsed-power discharge, to lasers.

The interaction of laser irradiation (or laser-induced X-ray radiation in a hohlraum) with matter may induce inertially confined high-pressure plasma and vapors. The pressure and reaction induce stress waves (e.g., shock wave) propagating into the sample - a versatile means of loading for the study of dynamic material properties [3, 4]. Research on material properties under extreme conditions utilizing laser-induced shock waves, though still developing, has demonstrated promise [5-11]. Here, we briefly review some experimental aspects in our efforts along this line, including the Trident laser facility at Los Alamos National Laboratory, some laser-loading techniques (laser-launched flyer plate and direct laser drive), diagnostics, and examples of their applications. Our focus is on demonstrating the

\footnotetext{
* Corresponding author. Fax: 1505665 3552; E-mail: sluo@lanl.gov
} 
suitability of laser shock experiments for acquiring useful data, rather than drawing specific scientific conclusions from the data.

\section{LASER AND LASER-INDUCED SHOCK WAVES}

Our laser shock wave experiments were mostly conducted at the Trident laser facility that includes a Nd:glass laser driver (fundamental wavelength $1054 \mathrm{~nm}$ ), target chambers, and optical/X-ray diagnostics. The laser driver employs an Nd:YLF master oscillator and a chain of $\mathrm{Nd}$ :phosphate glass rod and disk amplifiers in conventional master oscillator, power amplifier (MOPA) architecture. The oscillator pulse is temporally shaped, amplified and focused on target. Three beams $(\mathrm{A}-\mathrm{C})$ are available at the same or different frequencies of 1054, 527 and $351 \mathrm{~nm}$. Using a 13-element, intensity-variable pulse-stacker, the temporal shape, duration, and energy of laser pulses are adjustable for different experimental designs. Typical pulse shapes are triangular, Gaussian, square or stepped. The pulse durations can be varied from about 100 fs to $2 \mu$ s. Some illustrative loading pulses are shown in Figure 1. The maximum pulse energy (time-integrated) is about $500 \mathrm{~J}$ for $\mathrm{A}$ or B beam (for $1 \mu \mathrm{m}$ pulse of about $1 \mu \mathrm{s}$ duration) and $50 \mathrm{~J}$ for $\mathrm{C}$ beam. The laser spot size (diameter) of the illuminated area on the target surface can be varied between $\sim 50 \mu \mathrm{m}$ and $50 \mathrm{~mm}$, and phase plates are often used to smooth long-wavelength spatial variations in the beam. Diameters of about 1.5 and $5 \mathrm{~mm}$ for the laser spot are routinely adopted in our experiments.

Inertially confined high-pressure plasma and vapors are produced by rapid deposition of laser energy into matter surface; the pressure and reaction to expansion induce shock waves or quasi-isentropic ramp waves in condensed matter in direct laser drive experiments, or used to launched flyer plate (Fig. 2(a) and (b)). To launch a flyer plate, a pulsed laser is focused through a transparent substrate (PMMA, LiF or sapphire) onto a thin multilayer that has been deposited on the substrate (Fig. 2(a)). The multilayer is usually composed of a carbon layer to act as a working fluid and layers of aluminum and alumina to reduce lateral expansion of the plasma and heating of the flyer. A thin foil flyer plate, attached to the multilayer or substrate, has a diameter ranging from 1 to $8 \mathrm{~mm}$ and a thickness from

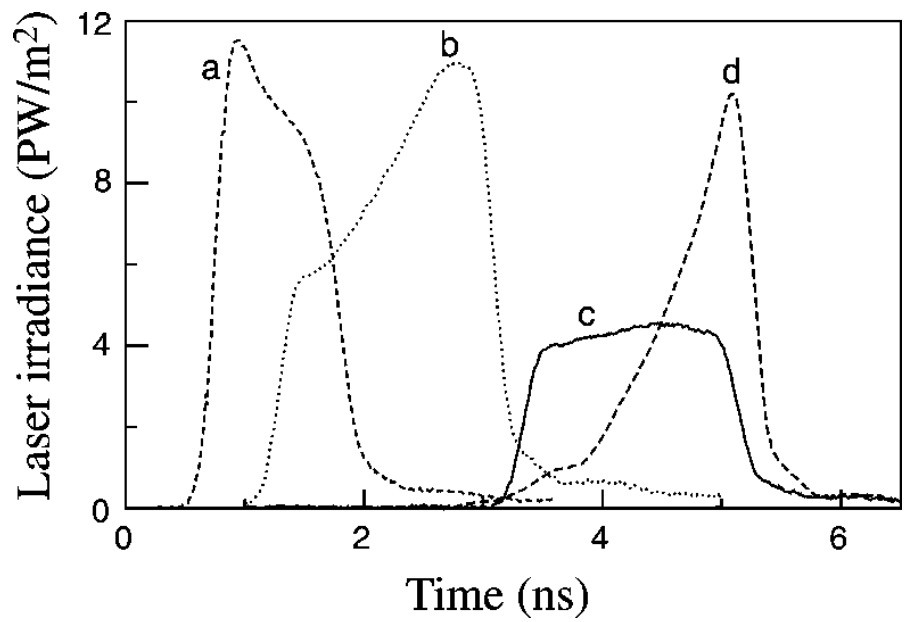

FIGURE 1 Laser drive-pulses of different shape, duration and irradiance ( $\mathrm{a}-\mathrm{d}$ are examples) inducing various loading (and unloading) stress waves. Pulses $\mathrm{b}$ and $\mathrm{d}$ are, in general, used for creating supported shock waves and ramp waves in direct laser-drive experiments, respectively. 
(a)

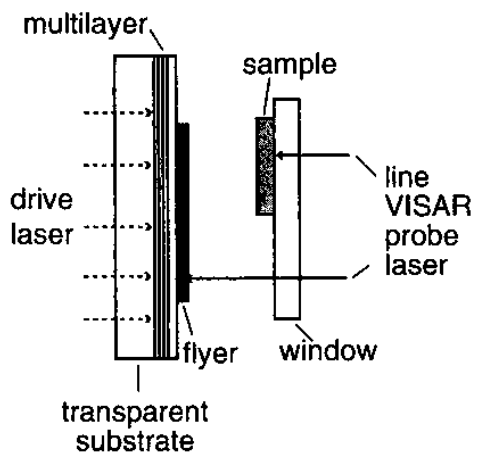

(b)

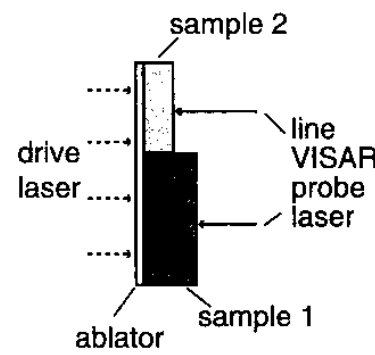

FIGURE 2 Schematic of laser shock wave experiments. (a) Laser-launched flyer plate. The multilayer is composed of (from left to right) carbon, Al, alumina, and Al layers. Line-imaging (or point) VISARs record the particle velocity histories of flyer and sample. (b) Direct laser drive. Laser shone on an ablator or directly on samples induces stress waves in two side-by-side samples (same or different). In both (a) and (b), the arrangement of window, samples, and transparent substrate can be varied to fit for equation of state and recovery experiments. For recovery, the whole assembly is kept in a brass cylinder.

about $5 \mu \mathrm{m}$ to $1 \mathrm{~mm}$. The laser pulse is absorbed in the carbon layer and creates a vapor or plasma that accelerates the flyer. The flyer acceleration is monitored with a point or lineimaging velocity interferometer system for any reflector (VISAR). The flight distance should be sufficient for achieving terminal velocity. For example, the $\mathrm{Cu}$ flyer (Fig. 3) barely reached its maximum speed before impact, although the effect of residual pressure behind the flyer was negligible.

State parameters such as pressure $(P)$ and density $(\rho)$ upon laser shock wave loading can be determined from flyer velocity $\left(u_{\mathrm{fp}}\right)$, shock velocity $\left(U_{\mathrm{s}}\right)$, or particle velocity $\left(u_{\mathrm{p}}\right)$, as well as equations of state of the flyer, target or window materials, just as in gas-gun experiments. For example, both $U_{\mathrm{s}}$ and $u_{\mathrm{p}}$ can be measured using stepped or wedged samples under wellcharacterized conditions. If insufficient measurements are available in direct laser drive, we can deduce the pressure in the ablator from an empirical pressure-irradiance relationship $[4,10,12]:$

$$
P \approx \alpha I^{\beta}
$$
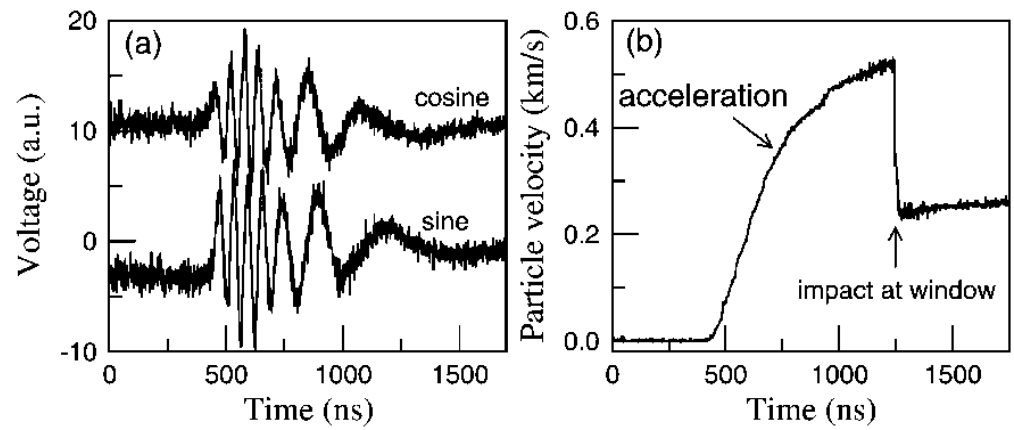

FIGURE 3 Laser-launched $\mathrm{Cu}$ flyer plate (thickness of $100 \mu \mathrm{m}$ and diameter $5 \mathrm{~mm}$ ) impacts sapphire window (Trident \#16040, laser energy $48 \mathrm{~J}$ at $527 \mathrm{~nm}$ ). (a) History of sine and cosine components of point-VISAR signal and (b) particle velocity of $\mathrm{Cu}$ flyer plate during acceleration and after impact at the window, reduced from (a). The uncertainty in velocity is about $\pm 5 \mathrm{~m} / \mathrm{s}$. 
where $P$ is in GPa and irradiance $I$ in $\mathrm{pW} / \mathrm{m}^{2} . \alpha$ and $\beta$ are material dependent. For most elements, $\beta$ is $2 / 3$ over a limited range of $I$ from radiation hydrodynamics simulations and experiments. For Al, we adopted $\alpha$ of 5.58 and $\beta$ of 0.77 [10] from fitting to radiation hydrodynamics calculations. The pressure in the sample can then be deduced by considering impedance mismatch.

The planarity of shock waves induced by lasers (as is the case for any shock wave generators) is critical for accurate determination of shock states using simple Rankine-Hugoniot jump conditions [2]. Besides sufficiently large ratio of laser-spot size to sample thickness, shock planarity also requires that the incident laser beam be spatially uniform. The planarity of loading is best demonstrated by temporally- and spatially-resolved measurement such as reflectivity and line-imaging VISAR measurement. The degree of non-planarity (bowing) of laser-launched flyer plate is typically 3-10 mrad. For example, reflectivity measurement on an A1 sample impacted by an A1 flyer plate demonstrate negligible bowing ( $2 \mathrm{mrad}$, Fig. 4(a)). The shock planarity can also be estimated from shock break-out in line-VISAR measurements (e.g., Fig. 4(b) for direct laser drive on a side-by-side NiAl assembly). The non-planarity of shock waves induced by direct laser drive is typically $10-60 \mathrm{mrad}$. The planarity and flyer plate integrity can be preserved to high velocity (e.g., $u_{\mathrm{fp}} \sim 7 \mathrm{~km} / \mathrm{s}$ or higher) in laser-launched flyer plate experiments. For direct-drive loading, the limit

\section{(a) Laser-launched flyer plate impact, reflecivity}

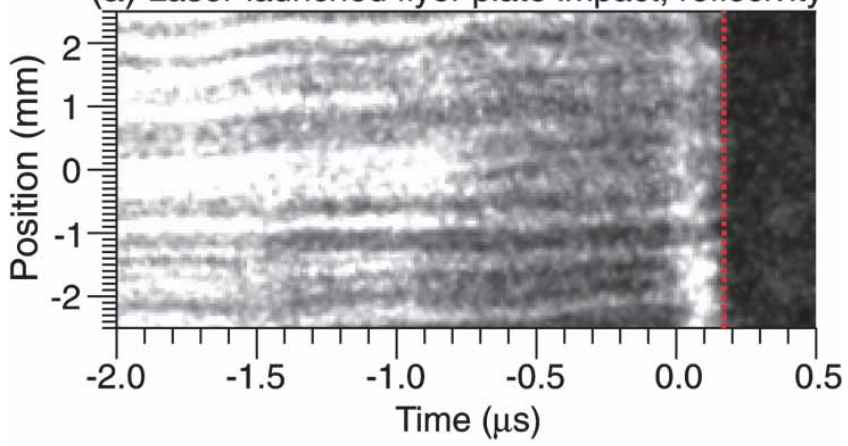

(b) Direct laser drive on NiAl, line-VISAR

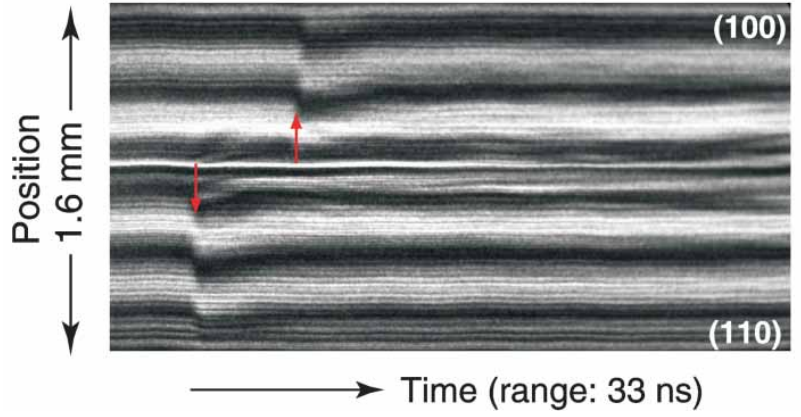

FIGURE 4 (a) Spatially- and temporally-resolved reflectivity measurement on Al sample (4 mm thick) impacted by a laser-launched Al flyer-plate (2 mm thick) (\#16831) before and after the impact (red dotted line) [13]. The velocity of the flyer at impact was about $330 \mathrm{~m} / \mathrm{s}$. (b) Line-VISAR record for direct laser drive on a side-byside NiAl assembly (\#15405): fringe displacement (corresponding to particle velocity) history at the back surface of NiAl crystals: $\left(\begin{array}{lll}1 & 0 & 0\end{array}\right)$ and $\left(\begin{array}{lll}1 & 1 & 0\end{array}\right)$ that were the same thickness. Shock break-out instants (arrows) indicate anisotropy in shock velocity of NiAl single crystal. One complete fringe displacement represents velocity of $800 \mathrm{~m} / \mathrm{s}[11]$. 
imposed by the integrity of flyer plate is absent, and planarity can be achieved in principle to much higher pressures. Besides, the flyer velocity is also limited by the breakdown energy of laser for the substrate. We often use approximately nanosecond pulses for ablation in direct drive experiments where sample thickness is about tens of $\mu \mathrm{m}$ if we want to see a non-decaying shock (not always the case). Then, the diameter-thickness ratio is typically huge; so, edge effects do not matter for many reverberation periods. We also intentionally limit our measurements to region on the sample where shock planarity is satisfactory. Another issue related is preheating of flyer plate and sample caused by fast electrons and $\mathrm{X}$-rays from laser-matter interactions. Previous studies have shown that preheating effect is negligible, up to at least 1 Mbar [10].

The flexibility of adjusting laser pulses allows both quasi-isentropic (ramp wave) and shock wave loading for probing different thermodynamic spaces. An isentrope can be obtained in a single ramp wave loading, whereas only one Hugoniot point is obtained from a shock wave experiment. For example, a ramp pulse (similar to pulse $d$ in Fig. 1) illuminated two side-by-side Fe foils of different thickness (respectively, 27 and $37 \mu \mathrm{m}$; setup similar to Fig. 2(b)), yielding two ramp stress waves (traces a and b, Fig. 5). The ramp duration in these two ramp stress waves is about $2.5 \mathrm{~ns}$, comparable to that in the loading pulse. Because of the thickness difference between these two foils, the ramp wave was observed only for the thinner foil (a in Fig. 5), whereas a shock seems to have developed from the ramp wave in the thicker foil (b in Fig. 5). In contrast, a shock was formed within about $0.5 \mathrm{~ns}$ when a pulse similar to $\mathrm{b}$ in Figure 1 was shone on an Fe foil $(22 \mu \mathrm{m})$, and the sustained shock lasted about $1.5 \mathrm{~ns}$ (c in Fig. 5). Ramp laser-pulse loading is a convenient way to study equation of state, complementing shock-wave loading.

A shock wave can be induced by lasers via direct drive, indirect hohlraum drive as well [6], or laser-launched flyer plate, broadening the range of states and spatial/temporal scales for studying mechanical properties such as plasticity and spallation, high-pressure physics and chemistry. The strain rates in laser shock waves vary from about $10^{6}$ to $10^{9} \mathrm{~s}^{-1}$. Shock durations, ranging from less than $100 \mathrm{ps}$ to more than $100 \mathrm{~ns}$, permit the evaluation of a wide range of materials on a microscale to a mesoscale (sub-grain size, single crystal to pseudo-bulk). In laser shock experiments, the instant at which pressure is

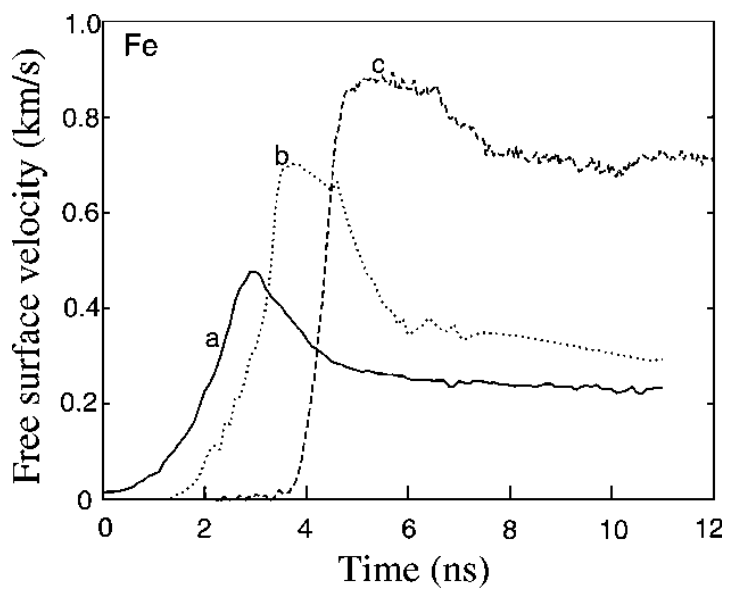

FIGURE 5 Free surface velocity history for direct laser-drive on iron: a ramp pulse on foils with thickness of $27 \mu \mathrm{m}$ (a) and $37 \mu \mathrm{m}$ (b), respectively. (Trident \#15413, laser $\lambda$ of $527 \mathrm{~nm}$, energy of $32 \mathrm{~J}$, and target assembled as side-by-side.) For comparison, Fe-foil $(22 \mu \mathrm{m})$ driven by a different pulse yielded a shock wave (c, time origin was shifted; Trident \#15395, laser $\lambda$ of $527 \mathrm{~nm}$, energy of $155 \mathrm{~J}$, laser spot size of about $5 \mathrm{~mm}$ ) [13]. 
generated is well defined by the arrival of laser pulse; thus, it is straightforward to synchronize shocks with a wide variety of optical, X-ray, and electronic diagnostics. By altering the laser energy and focal spot on the sample, the pressure induced by direct drive can be varied in a controllable way from less than about $1 \mathrm{GPa}$ to at least $1 \mathrm{TPa}$ with a shock duration of less than $100 \mathrm{~ns}$. Laser-launched flyer plates can generate shocks with pressure up to several megabars and duration ranging from less than $1 \mathrm{~ns}$ to more than $100 \mathrm{~ns}$, bridging the gap between direct laser drive and gas gun loading where pressure is limited to about $2 \mathrm{Mbar}$ and shock duration longer than $100 \mathrm{~ns}$. Unlike the more conventional gas gun and highexplosive methods, as well as pulsed-power discharge such as a Z-pinch facility, there is less stray energy or momentum for laser-induced shocks around the experiment from the sabot, detonation products, and so on. This makes it easier to recover and interpret the microstructure of the shocked sample.

\section{DIAGNOSTICS AND APPLICATIONS}

\section{Velocity Interferometry}

The VISAR is widely used in dynamic experiments for measuring particle velocity [14-17]. A probe laser ( $660 \mathrm{~nm}$ for line-VISAR and $532 \mathrm{~nm}$ for point VISAR, in our experiments) is reflected from the moving surface of a shocked sample, and its frequency is shifted due to the Doppler effect. The frequency-shifted laser beam enters an interferometer that produces temporally-resolved (and spatially-resolved for line-VISAR) fringe displacements. Point and line VISAR signals are recorded by photomultipliers and electronic streak cameras, respectively. Examples of fringe patterns and velocity histories reduced from them for these two types of VISAR are shown in Figures 3-5. Line-VISAR signals not only have high temporal resolutions (100 ps order), but also measure the planarity of flyer plates and shock waves. Line-VISAR fringes also allow the shock wave velocity to be determined from the shock break-out, if stepped or wedged samples are used (similar to Fig. 2(b)). If different samples of the same thickness are used in a side-by-side target assembly, the difference in shock wave velocity for these two samples can be investigated. For example, the two $\mathrm{NiAl}$ single crystals with the same thicknesses but different orientations were subjected to direct laser drive. The shock break-out occurred at distinct instants, indicating the anisotropy of shock wave velocity in single-crystal NiAl (Fig. 4(b)). The EOS can be deduced from measured $U_{\mathrm{s}}$ or $u_{\mathrm{p}}$, as well as $u_{\mathrm{fp}}$, for flyer plate.

In laser-launched flyer-plate loading $[9,18], u_{\mathrm{fp}}$ and $u_{\mathrm{p}}$ at the flyer-window interface during impact can be measured from a single shot (Fig. 3). As the windows are standard materials with known EOS, the shock state can be deduced from $u_{\mathrm{fp}}$ and $u_{\mathrm{p}}$ via the impedance mismatch technique. We use copper as a test material for validation of EOS measurement using laser-launched flyer-plate loading and for investigation of spallation at different strain rates, both readily compared to gas or powder gun data. We found that the $U_{\mathrm{s}}-u_{\mathrm{p}}$ pairs from laser-flyer-plate experiments were in excellent agreement with the gun data (Fig. 6). Although our experiments were intentionally focused on the low pressure end, we expect that with smaller flyer plate and higher irradiance, much higher pressure (mega bar order) can be achieved with accuracy comparable with gas-gun data.

Spallation of metals, ceramics, and other solids are of considerable interest for their engineering applications and for understanding the physics and mechanics of spallation itself [18, 21-25]. Spallation occurs when the tensile stress exceeds the spall strength of a material for a given strain rate. In our experiments, the flyer and target are usually the same materials, and the ratio of their thicknesses is about $1: 2$. Thus, spallation occurs near the mid-plane of the 


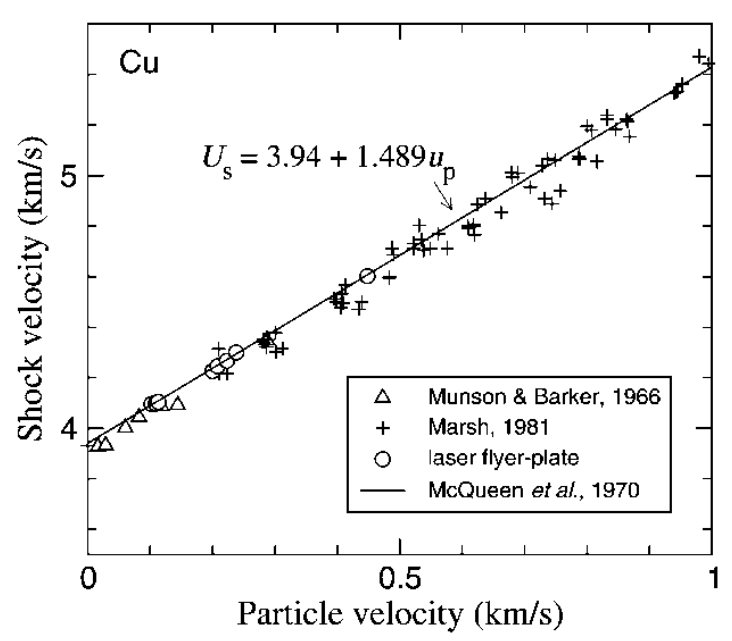

FIGURE 6 Equation of state $\left(U_{\mathrm{s}}-u_{\mathrm{p}}\right)$ of Cu from laser-flyer-plate experiments (this work and Ref. [18]) compared with gun experiments $[2,19,20]$.

target. The tensile stress is induced by two colliding release waves (from the free surfaces of the sample and flyer plate). The strain rates $(\dot{\varepsilon})$ achievable in traditional gas gun and Hopkinson bar loading can barely exceed $10^{6} \mathrm{~s}^{-1}$. The strain rates achievable in laser-flyer-plate experiments overlap with gas gun and can reach much higher values $\left(10^{5}-10^{9} \mathrm{~s}^{-1}\right)$. For the same material, different $(\dot{\varepsilon})$ can be achieved by varying the thickness of target and flyer. A thinner target will have higher pressure-gradient at its mid-plane than a thicker one if impacted at the same velocity. As the pressure-gradient relates directly to strain rate, we expect that thinner flyer-target assemblies will produce higher strain rate at given impact velocity or shock pressure. Spallation can be conveniently investigated from the free surface velocity history of a shocked sample - a representative profile for high purity tin (Fig. 7(a)) shows the shock, release, spallation, and pull-back.
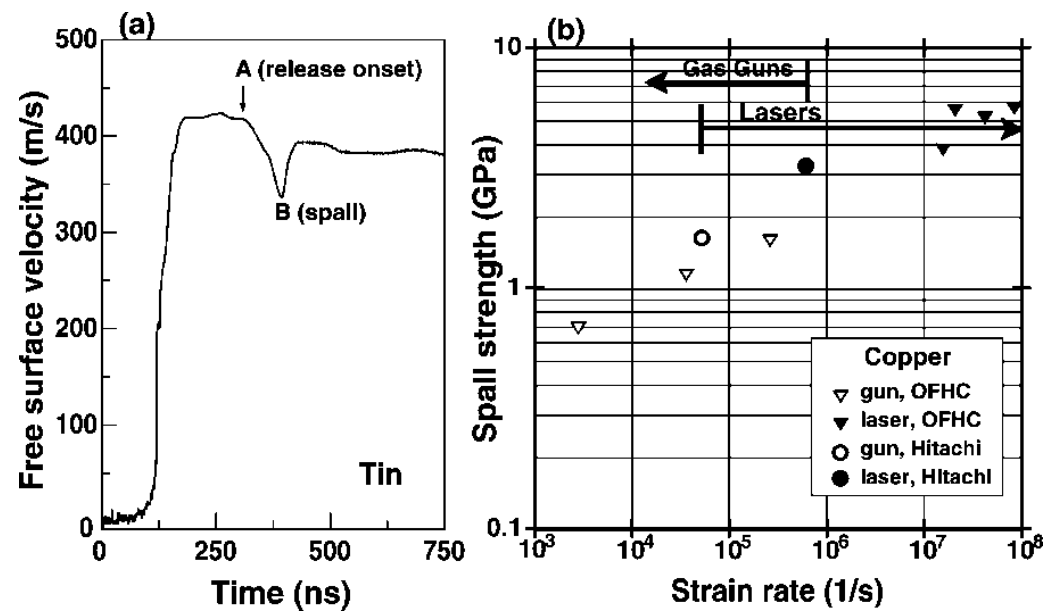

FIGURE 7 Spall strength versus strain rates [26]: (a) typical free surface velocity history showing release onset (A), spallation (B), and subsequent pull-back for tin (Trident \#16062); (b) comparison between laser launched flyer plate experiments with gas-gun data for two types of $\mathrm{Cu}$, OFHC and Hitachi. 
The strain rate $(\dot{\varepsilon})$ upon release within the acoustic approximation [22] is

$$
\dot{\varepsilon} \approx \frac{1}{C_{0}} \frac{\mathrm{d} u_{\mathrm{p}}}{\mathrm{d} t} \approx \frac{1}{2 C_{0}} \frac{u_{\mathrm{A}}-u_{\mathrm{B}}}{t_{\mathrm{B}}-t_{\mathrm{A}}}
$$

where $C_{0}$ is the bulk sound speed at ambient conditions and $u$ is the free surface velocity. Subscripts A and B are referred in Figure 7(a). For example, for Trident flyer-plate shot \#16062 on high-purity tin with $C_{0}=2.747 \mathrm{~km} / \mathrm{s}$ [20] (Fig. 7(a)), we have $\dot{\varepsilon} \approx 2.6 \times 10^{5} \mathrm{~s}^{-1}$. The spall strength $\sigma_{\mathrm{sp}}$ within the acoustic approximation is

$$
\sigma_{\mathrm{sp}} \approx \frac{1}{2} \rho_{0} C_{0}\left(u_{\mathrm{A}}-u_{\mathrm{B}}\right)
$$

where $\rho_{0}$ is ambient density. In the case of tin (\#16062), $\rho_{0}=7.31 \mathrm{~g} / \mathrm{cm}^{3}$, and $\sigma_{\mathrm{sp}} \approx 0.8 \mathrm{GPa}$ at $\dot{\varepsilon} \approx 2.6 \times 10^{5} \mathrm{~s}^{-1}$ (depending on the models adopted, a correction term to $\sigma_{\mathrm{sp}}$ may be necessary for Eq. (3) [25]).

Experiments similar to tin were conducted using laser-launched flyer plate on oxygen-free high-conductivity (OFHC) copper and Hitachi copper (some of these experiments were conducted on a smaller laser system at Los Alamos). The spall strength as a function of strain rate from laser experiments was compared to gas-gun experiments on the same materials (Fig. 7(b)). For OFHC-type $\mathrm{Cu}$, both the low strain-rate gas gun data and the high strainrate laser flyer-plate data can be described by a single power law: $\sigma_{\mathrm{sp}} \propto \dot{\varepsilon}^{n}$, where $n \approx 0.2$ the same as given by Antoun et al. [25]. Because of its higher purity, the Hitachi copper demonstrates slightly higher $\sigma_{\mathrm{sp}}$ than OFHC copper (Fig. 7).

Spallation also occurs upon direct laser drive - the spallation mechanism for some loading pulses is similar to that upon flyer plate loading. For decaying pulse loading (pulse a in Fig. 1), spallation can be caused by the interaction of the release wave from sample free surface with the tail of loading stress wave [22]. Investigations on spallation by direct laser drive with $\dot{\varepsilon} \sim 10^{8} \mathrm{~s}^{-1}$ or higher have been reported [24].

\section{Transient X-ray Diffraction}

Transient X-ray diffraction (TXD), i.e., time-resolved X-ray diffraction from the shocked lattice, has great potential for investigating solid-solid and solid-liquid phase transitions and elastic-plastic behavior of shock loaded samples [12, 27-35, 38]. In earlier powderor gas-gun shock wave experiments, Zavada et al. [27] and Johnson and Mitchell [28] reported real-time X-ray diffraction during shock compression. Rigg and Gupta [33] reported quantitative elastic-plastic behavior of shocked LiF using a similar technique where the real-time diffraction pattern was time-integrated over the pulse duration of the X-ray source. Similar experiments have been conducted at nanosecond-scale using laser shocks. TXD results have been reported on single-crystal and polycrystalline solids (e.g., InSb, $\mathrm{Ge}, \mathrm{Si}, \mathrm{Cu}$, and $\mathrm{Be}$ ) subjected to laser-induced shock waves [31, 32, 35-37].

The setup for TXD simultaneously with VISAR at Trident is shown in Figure 8 [29]. The $\mathrm{X}$-rays are generated by focusing a second laser beam at high energy and $527 \mathrm{~nm}$ (green) tightly (about $100 \mu \mathrm{m}$ spot) onto a metallic foil (e.g., $\mathrm{Mg}$, Ti, and $\mathrm{Mn}$ ) in a gold cone. The resulting hot plasma emits helium- $\alpha$ X-rays with certain characteristic wavelengths for each element and can be used for diffraction. The TXD patterns can be recorded by $\mathrm{X}$-ray streak cameras (denoted as Laue and Bragg, Fig. 8). Time-integrated diffraction signals can also be recorded using film or image plate. Meanwhile, the VISAR fringe displacements supply particle velocity history (e.g., Figs. 3-5) for deducing shock states. The 


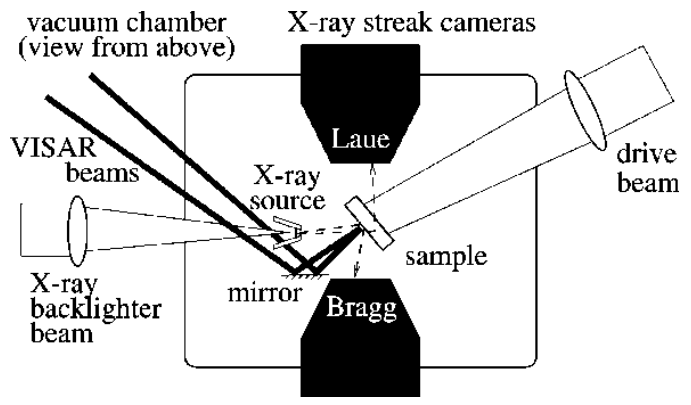

FIGURE 8 Schematic representation of laser irradiation with TXD and VISAR diagnostics at Trident. A laser beam shone on the sample or ablator induces shock waves propagating inside. TXD patterns are recorded at two perpendicular directions by X-ray streak cameras (denoted as Laue and Bragg).

change of lattice spacing $(\Delta d)$ induced by shock compression gives rise to a change in Bragg angle $\theta$ with respect to unshocked lattice, and they are related as [32] $\Delta \theta=-(\Delta d / d) \tan \theta$. Note that to relate $\Delta d / d$ to volume changes from EOS, knowledge of the nature of compression (uniaxial or hydrostatic) is necessary [33]. This can be obtained by simultaneous diffraction measurements at multiple places at Trident.

As an example, Figure 9 shows TXD patterns recorded by the Bragg camera from a silicon single crystal subjected to direct laser drive along [ $\left[\begin{array}{lll}0 & 0 & 1\end{array}\right]$ direction [35]. The recorded signal is the integral of diffraction from the lattices along the X-ray penetration depth in the sample, thus containing signals of both shocked and unshocked lattices. In Figure 9, portions a and b represent diffraction from unshocked lattice, $c$ from shocked lattice, and $d$ from relaxation of shocked lattice. At higher pressures (e.g., $20 \mathrm{GPa}$ ), splitting of Bragg line indicates possible solid-solid phase transition from the diamond to body-centered tetragonal (bct) structure. Our preliminary TXD measurements on gallium indicated melting transition upon direct laser drive [38].

Besides phase transitions, the current TXD setup has been used to study elastic-plastic behavior [31]. Such experiments on single crystal $\mathrm{Si}$ and $\mathrm{Cu}$ revealed distinct behavior: for $\mathrm{Si}\left(\begin{array}{ll}0 & 0\end{array}\right)$, the compression was uniaxial even when the lattice spacing was reduced by $11 \%$, whereas shocked $\mathrm{Cu}$ demonstrated prompt hydrostatic-like compression. This difference was related to the dislocation mobilities for $\mathrm{Si}$ and $\mathrm{Cu}$ [31]. Another interesting issue similar to LiF, i.e., the anisotropy of elastic-plastic behavior [33], can also be investigated through our TXD studies.

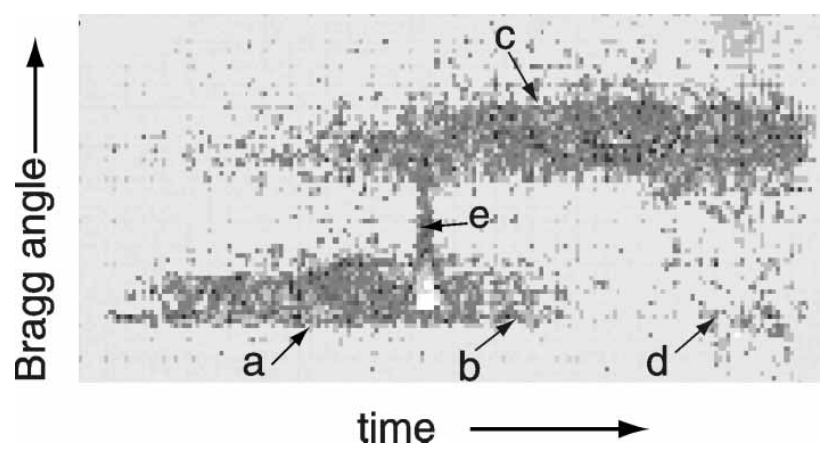

FIGURE 9 Representative TXD record (5 ns duration) on Si single crystal shocked along [0 0 1]. Diffractions from both shocked (c) and unshocked ( $a$ and $b$ ) lattices were recorded. Portion e was formed from direct X-ray shining through the slit in the streak camera and portion $\mathrm{d}$ from relaxed lattice upon release of pressure [35]. 


\section{Spectroscopy}

We broadly include ellipsometry, Raman, and shock temperature measurement in spectroscopic techniques. Optical properties, such as polarization and emissivity (both can be related to the complex refractive index [39]), and vibrational spectra depend on crystal structure and thermodynamic states. All these techniques have been adopted in the study of dynamic material properties [40-45]. For example, time-resolved Raman measurements were conducted on shocked $\alpha$-quartz to $6 \mathrm{GPa}$ using a powder gun and on molecular solids from laser shocks [41]. Temperatures and emissivities of gas-gun loaded translucent materials were determined from time-resolved radiation recorded by a multichannel pyrometer [45]. Reflectivity and optical brightness of laser-shocked silicon were reported under extreme compression (4-8 Mbar) [44].

These spectroscopic techniques attempted to investigate the shock behavior of opaque materials under laser-induced shocks at Trident. The inherent technical difficulties in spectroscopic measurements on these materials limit our measurements to the free surface of a sample or to the sample-window interface; so, the measurements represent (partially) released or reshocked states. As an example, we attempted to resolve solid-solid and solid-liquid transitions from the time-resolved ellipsometry measurements on $\mathrm{Si}$ and $\mathrm{Sn}$ upon direct laser drive. A depolarized laser beam was reflected from the sample surface at a glancing angle, through a release window. The intensity of $\mathrm{S}$ and $\mathrm{P}$ polarized light in the reflected signal was recorded using a streak camera, containing information on the optical properties of shocked samples. Their dramatic changes at certain pressures are reasonable indicators of phase transitions. As shown in Figure 10, the distinct behaviors of $\mathrm{S}$ and $\mathrm{P}$ polarizations at two different pressures may indicate that the diamond to bet transition in Si has occurred. Similarly, the distinct behaviors of both $\mathrm{S}$ and $\mathrm{P}$ polarizations reflected from the tin- $\mathrm{LiF}$ interface at about 8 and $28 \mathrm{GPa}$ may indicate the melting of tin. We use only ellipsometry as a complement to other techniques in studying phase changes, and caution should be taken, as some factors other than phase changes may also affect the ellipsometry measurements.

\section{Recovery}

Lasers deliver energy with negligible momentum, which is advantageous for shock recovery. For laser-launched flyer-plates, recovery of (spalled) samples is possible even at strain rates as high as $10^{8} \mathrm{~s}^{-1}$. Recovered samples are then post-examined with various analytical
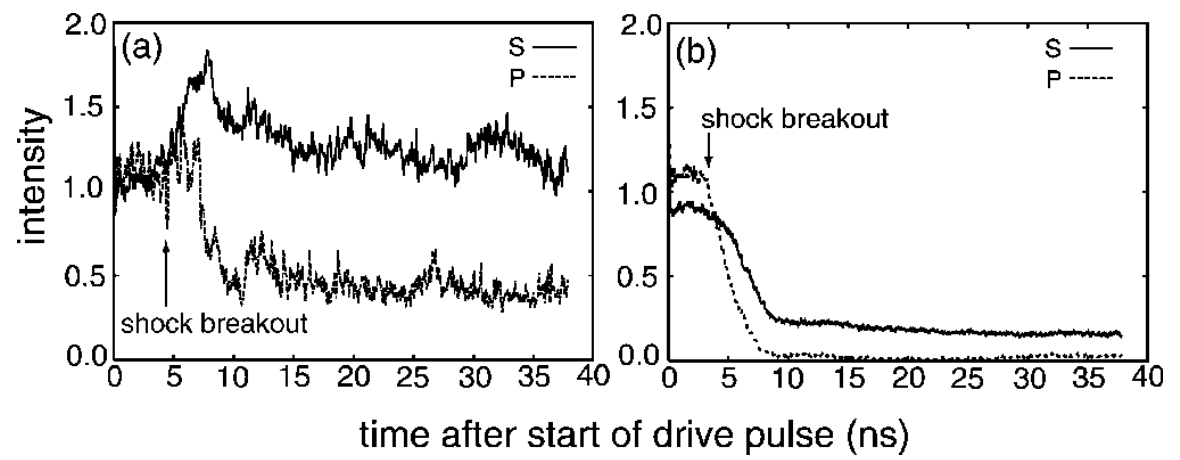

FIGURE 10 Ellipsometry signals recorded at the interface of Si ( 1000$)$ and window [37]: (a) below diamond-bct transition (Trident \#14968, $P \sim 9 \mathrm{GPa}$ ) and (b) around diamond-bct transition (Trident \#14969, $P \sim 14-17 \mathrm{GPa}$ ). 
techniques to investigate the physical, mechanical, and chemical effects of loading, including metallography [46] and Raman [47], X-ray, and neutron scattering. Although laser shock recovery has been extensively conducted at Trident (e.g., on $\mathrm{Al}, \mathrm{Cu}, \mathrm{Ti}, \mathrm{Si}, \mathrm{NiTi}, \mathrm{NiAl}$, $\mathrm{SiO}_{2}, \mathrm{MgB}_{2}$, and $\mathrm{C}$ ), here we only discuss silica and carbon. Silica and carbon are wellstudied prototype materials rich in polymorphs and phase-transition physics including kinetics caused by the extremely high heating and compression rates accessible to laser experiments.

In silica and carbon recovery experiments with direct laser drive, we used $\mathrm{Al}$ foil as an ablator and an LiF window. In principle, shock states can be determined from VISAR measurements as discussed earlier. In the preliminary experiments reported here, VISAR signal levels were poor due to the poor reflectivity from these diffuse samples. Instead, we deduced the supported pressure in the Al ablator using Eq. (1) and determined the shock states from the EOS of $\mathrm{Al}$ and sample by taking into account the impedance match. In the case of mixture, the EOS (hence the Hugoniot) was obtained from the complete EOS of each component.

Single-crystal $\alpha$-quartz was subjected to laser loading with a $12.5 \mu \mathrm{m} \mathrm{Al}$ ablator (Trident \#16466). The $527 \mathrm{~nm}$ laser passing through a Fresnel zone plate formed a spot of $1.5 \mathrm{~mm}$ in diameter on the ablator. The laser pulse had a $2.4 \mathrm{~ns}$ duration and an energy of $123 \mathrm{~J}$. The sustained pressure in the shocked quartz was about $54 \mathrm{GPa}$, close to previous powder-gun recovery experiments on quartz and coesite [48]. The recovered sample was examined with Raman spectroscopy. Although the examined portion was predominantly quartz, we observed changes in the Raman spectrum, indicating structural disorder characteristic of the onset of a coordination change in silica. Similar effects on the Raman spectrum of quartz had been found in recent laser shock experiments [47] which also indicate the transformation of quartz into a transitional phase observed in powder-gun experiments [48]. We plan to conduct micro-Laue diffraction on laser-shocked quartz crystallites in order to acquire detailed information on intermediate structures, as well as the transformation path from four- to six-fold coordinated silica.

Laser shock recovery experiments were conducted on binary mixtures of metals $(\mathrm{Cu}$ and Ti) and graphite (or $\left.\mathrm{C}_{60}\right)$ at the Trident laser facility. Pellets of sample materials $(\sim 0.5 \mathrm{~mm}$ thick), sandwiched between an LiF window and an Al ablator (12.5 $\mu \mathrm{m}$ thick), were subjected to shock wave loading from the ablator irradiated by a laser pulse of $\sim 2.4 \mathrm{~ns}$ duration and $527 \mathrm{~nm}$ wavelength (Fig. 11). The laser-spot size illuminating the ablator was about $5 \mathrm{~mm}$. The recovered samples were examined by synchrotron X-ray diffraction and Raman scattering. Although shock experiments on carbon phases usually yield mixtures of poorly crystallized allotropes, we found several well crystallized phases in the samples recovered from laser-shock experiments. Monochromatic synchrotron radiation was
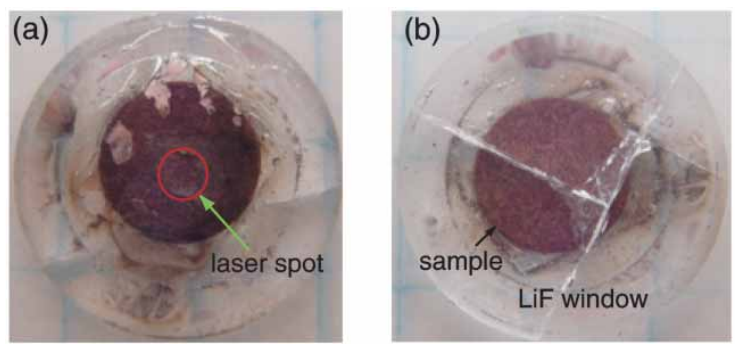

FIGURE 11 Recovery experiment on graphite-Cu mixture (Trident \#16726). The diameter of the window is about $14 \mathrm{~mm}$ : (a) front side (laser ablator); (b) back side ( $\mathrm{LiF}$ window). 


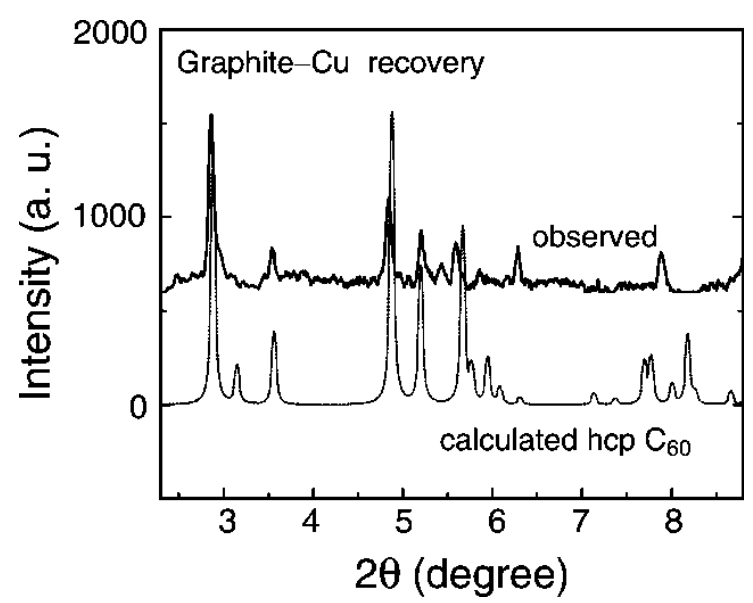

FIGURE 12 Synchrotron X-ray diffraction pattern (refined) of shocked graphite-Cu mixture (Trident \#16726), indicating a hexagonal crystalline phase of two-dimensional polymerization.

focused on the sample (beam diameter of $100 \mu \mathrm{m}$ ), and diffracted X-rays were collected with an image-plate detector. Here, we show as an example the pattern of a graphite-copper mixture after shocking (shot \#16726, laser energy $185 \mathrm{~J}$, and pressure about $15 \mathrm{GPa}$ ). The close match between observed and calculated Bragg angles (Fig. 12) indicates the production of well-crystallized $\mathrm{C}_{60}$-fullerite, and no residual graphite is observed. Intensities and splitting of the Bragg peaks at low angles are consistent with a pseudo-hexagonal twodimensional (2D) polymeric fullerite. There are distinct differences compared with the 2D hexagonal fullerite retrieved from static compression experiments. Smaller portions of the sample indicate orthorhombic 1D fullerite. The narrow peak width and high intensity of the Bragg peaks indicate a surprisingly high degree of crystalline order. The laser-shocked Ti-graphite mixture (shot \#16765, laser energy $204 \mathrm{~J}$, and pressure about $14 \mathrm{GPa}$ ) yielded carbon structures possibly with three-fold coordinated $\mathrm{C}$-atoms, similar to the prediction by O'Keeffe et al. [49].

\section{CONCLUSION}

We have reviewed some techniques of laser-induced shock waves including direct laser drive and laser-launched flyer plates, with optical and X-ray diagnostics, and their applications in the study of high-pressure physics and dynamic material properties. The developing laserinduced shock wave techniques have great potential in the research of dynamic material properties as a complement to conventional shock wave techniques.

\section{Acknowledgements}

One of the authors (S.N.L.) is sponsored by a Director's Postdoctoral Fellowship at LANL. The authors are grateful for the invaluable support from the Trident laser facility and staff at LANL. O.T. acknowledges support by the NNSA Cooperative Agreement DE-FC8801NV14049. Use of the HPCAT facility was supported by DOE-BES, DOE-NNSA, NSF, DOD-TACOM, and the W.M. Keck Foundation. This work was performed in part under the auspices of US Department of Energy under contract \#W-7405-ENG-36. 


\section{References}

[1] H. K. Mao and R. J. Hemley, New windows on earth and planetary interiors, Mineral. Mag., 66, 791 (2002).

[2] R. G. McQueen, S. P. Marsh, J. Q. Taylor, J. N. Fritz and W. J. Carter, The equation of state of solids from shock wave studies, In: R. Kinslow (Ed.), High-Velocity Impact Phenomena (Academic Press, New York, 1970), pp. 293-417.

[3] C. G. M. van Kessel and R. Sigel, Observation of laser-driven shock waves in solid hydrogen, Phys. Rev. Lett., 33, 1020 (1974).

[4] T. J. Trainor and Y. T. Lee, Analytical models for design of laser-generated shock-wave experiments, Phys. Fluids, 25, 1898 (1982).

[5] A. Ng, Shock waves and plasma physics, In: M. D. Furnish, N. N. Thadhani and Y. Horie (Eds.), Shock Compression of Condensed Matter - 2001 (AIP Conf. Proc., CP620) (Am. Inst. Phys., Melville, 2002), pp. 53-58.

[6] S. D. Rothman, A. M. Evans, R. T. Eagleton and L. B. Pearson, Material properties experiments using the AWE high power laser, Helen, Int. J. Impact Eng., 23, 803 (1999).

[7] I. K. Krasyuk, P. P. Pashinin, A. Y. Semenov and V. E. Fortov, Investigation of thermophysical and mechanical properties of matter under extremal conditions, Quantum Electron., 33, 593 (2003).

[8] J. P. Chen, R. X. Li, Z. N. Zeng, X. T. Wang, W. Y. Wang, Y. H. Jiang, C. F. Cheng and Z. Z. Xu, Simultaneous measurement of laser-induced shock wave and released particle velocities at Mbar pressure, J. Appl. Phys., 94, 858 (2003).

[9] D. L. Paisley, R. H. Warnes and R. A. Kopp, Laser-driven flat plate impacts to $100 \mathrm{GPa}$ with sub-nanosecond pulse duration and resolution for material property studies, In: S. C. Schmidt, R. D. Dick, J. W. Forbes and D. G. Tasker (Eds.), Shock Compression of Condensed Matter - 1991 (North-Holland, Amsterdam, 1992), pp. $825-828$.

[10] D. C. Swift, T. E. Tierney IV, R. A. Kopp and J. T. Gammel, Shock pressures induced in condensed matter by laser ablation, Phys. Rev., E69, 36406 (2004).

[11] D. C. Swift, J. T. Gammel and S. M. Clegg, Treatment of compounds and alloys in radiation hydrodynamics simulations of ablative laser loading, Phys. Rev., E69, 056401 (2004).

[12] A. A. Hauer, J. S. Wark, D. Kalantar, B. Remington, R. Kopp, J. Cobble, B. Failor, G. Kyrala, M. Meyers, R. Springer and T. Boehley, Transient x-ray diffraction and its applications to materials science and X-ray optics, Proc. SPIE, 3157, 72 (1997).

[13] T. E. Tierney, Personal communication (2004).

[14] L. M. Barker, The development of the VISAR, and its use in shock compression science, In: M. D. Furnish, L. C. Chhabildas and R. S. Hixson (Eds.), Shock Compression of Condensed Matter - 1999 (AIP Conf. Proc., CP505) (Am. Inst. Phys., Melville, 2000), pp. 11-17.

[15] L. M. Barker and R. E. Hollenback, Laser interferometer for measuring high velocities of any reflecting surface, J. Appl. Phys., 43, 4669 (1972).

[16] W. F. Hemsing, A. R. Mathews, R. H. Warnes and G. R. Whittemore, Line-imaging interferometer, In: L. L. Shaw, P. A. Jaanimagi, B. T. Neyer (Eds.), Ultrahigh- and High-speed Photography, Videography, Photonics, and Velocimetry (Proc. SPIE, 1346), 1990, pp. 133-140.

[17] A. C. Forsman and G. A. Kyrala, Non-Doppler shift related experimental shock wave measurements using velocity interferometer systems for any reflector, Phys. Rev., E63, 056402 (2001).

[18] R. H. Warnes, D. L. Paisley and D. L. Tonks, Hugoniot and spall data from the laser-driven miniflyer, In: S. C. Schmidt and W. C. Tao (Eds.), Shock Compression of Condensed Matter - 1995 (AIP Conf., Proc. CP370) (Am. Inst. Phys., Melville, 1996), pp. 495-498.

[19] D. E. Munson and L. M. Barker, Dynamically determined pressure-volume relationships for aluminum, copper, and lead, J. Appl. Phys., 37, 1652 (1966).

[20] S. P. Marsh, LASL Shock Hugoniot Data (University of California Press, Berkeley, 1981).

[21] D. E. Grady and M. E. Kipp, Dynamic fracture and fragmentation, In: J. R. Asay and M. Shahinpoor (Eds.), High-Pressure Shock Compression of Solids (Springer-Verlag, New York, 1992), pp. 265-322.

[22] E. Dekel, S. Eliezer, Z. Henis, E. Moshe, A. Ludmirsky and I. B. Goldberg, Spallation model for the high strain rates range, J. Appl. Phys., 84, 4851 (1998).

[23] V. E. Fortov, D. Batani, A. V. Kilpio, I. K. Krasyuk, I. V. Lomonosov, P. P. Pashinin, E. V. Shashkov, A. Yu. Semenov and V. I. Vovchenko, The spall strength limit of matter at ultrahigh strain rates induced by laser shock waves, Laser Part. Beams, 20, 317 (2002).

[24] E. Moshe, S. Eliezer, Z. Henis, M. Werdiger, E. Dekel, Y. Horovitz, S. Maman, I. B. Goldberg and D. Eliezer, Experimental measurements of the strength of metals approaching the theoretical limit predicted by the equation of state, Appl. Phys. Lett., 76, 1555 (2000).

[25] T. Antoun, L. Seaman, D. R. Curran, G. I. Kanel, S. V. Razorenov and A. V. Utkin, Spall Fracture (SpringerVerlag, New York, 2003).

[26] D. L. Paisley, Personal communication (2004).

[27] N. I. Zavada, M. A. Manakova and V. A. Tsukerman, Recording of interferences of monocrystals and polycrystals with microsecond exposures, Pribory i Tekhnika Eksperimenta, 2, 164 (1966) (English translation, Instruments and Experimental Techniques, 2, 434).

[28] Q. Johnson and A. C. Mitchell, First X-ray diffraction evidence for a phase transition during shock-wave compression, Phys. Rev. Lett., 29, 1369 (1972). 
[29] A. A. Hauer and G. A. Kyrala, Laser-plasma x-ray emission: its creation, diagnosis, and application in transient diffraction, In: J. R. Helliwell and P. M. Rentzepis (Eds.), Time Resolved Difiaction (Oxford University Press, New York, 1997), pp. 71-105.

[30] R. R. Whitlock and J. S. Wark, X-ray diffraction dynamics of shock compressed crystals, In: J. R. Helliwell and P. M. Rentzepis (Eds.), Time Resolved Diffraction (Oxford University Press, New York, 1997), pp. 106-136.

[31] A. Loveridge-Smith, A. Allen, J. Belak, T. Boehly, A. Hauer, B. Holian, D. Kalantar, G. Kyrala, R. W. Lee, P. Lomdahl, M. A. Meyers, D. Paisley, S. Pollaine, B. Remington, D. C. Swift, S. Weber and J. S. Wark, Anomalous elastic response of silicon to uniaxial shock compression on nanosecond time scales, Phys. Rev. Lett., 86, 2349 (2001).

[32] J. S. Wark, R. R. Whitlock, A. A. Hauer, J. E. Swain and P. J. Solone, Subnanosecond x-ray diffraction from laser-shocked crystals, Phys. Rev., B40, 5705 (1989).

[33] P. A. Rigg and Y. M. Gupta, Real-time x-ray diffraction to examine elastic-plastic deformation in shocked lithium fluoride crystals, Appl. Phys. Lett., 73, 1655 (1998).

[34] E. B. Zaretskii, G. I. Kanel, P. A. Mogilevshii and V. E. Fortov, Device for investigating x-ray diffraction on shock-compressed material, High Temp., 29, 805 (1991).

[35] D. C. Swift, G. J. Ackland, A. Hauer and G. A. Kyrala, First-principles equations of state for simulations of shock waves in silicon, Phys. Rev., B64, 214107 (2001).

[36] D. C. Swift, D. L. Paisley, G. A. Kyrala and A. A. Hauer, Simultaneous VISAR and TXD measurements on shocks in beryllium crystals, In: M. D. Furnish, N. N. Thadhani and Y. Horie (Eds.), Shock Compression of Condensed Matter - 2001 (AIP Conf. Proc., CP620) (Am. Inst. Phys., Melville, 2002), pp. 1192-1195.

[37] D. C. Swift, Personal communication (2004).

[38] S. N. Luo, D. C. Swift, T. Tierney, K. Xia, O. Tschauner and P. D. Asimow, Time-resolved x-ray diffraction investigation of superheating-melting behavior of crystals under ultrafast heating, In: M. D. Furnish, Y. M. Gupta and J. W. Forbes (Eds.), Shock Compression of Condensed Matter - 2003 (AIP Conf. Proc., CP706) (Am. Inst. Phys., Melville, 2004), pp. 95-98.

[39] G. Pottlacher and A. Seifter, Microsecond laser polarimetry for emissivity measurements on liquid metals at high temperatures - application to tantalum, Int. J. Thermophys., 23, 1281 (2002).

[40] A. W. Obst, K. R. Alrick, W. W. Anderson, K. Boboridis, W. T. Buttler, S. K. Lamoreaux, B. R. Marshall, S. L. Montgomery, J. R. Payton and M. D. Wilke, Ellipsometry in the study of dynamic material properties, In: M. D. Furnish, N. N. Thadhani and Y. Horie (Eds.), Shock Compression of Condensed Matter - 2001 (AIP Conf. Proc., CP620) (Am. Inst. Phys., Melville, 2002), pp. 1247-1250.

[41] R. L. Gustavsen and Y. M. Gupta, Time resolved Raman measurements in $\alpha$-quartz shocked to 60 kbar, J. Appl. Phys., 75, 2837 (1994).

[42] D. S. Moore, S. C. Schmidt, M. S. Shaw and J. D. Johnson, Coherent anti-Stokes Raman spectroscopy of shockcompressed liquid carbon monoxide, J. Chem. Phys., 95, 5603 (1991).

[43] S. A. Hambir, J. Franken, D. E. Hare, E. L. Chronister, B. J. Baer and D. D. Dlott, Ultrahigh time-resolution vibrational spectroscopy of shocked molecular solids, J. Appl. Phys., 81, 2157 (1997).

[44] Th. Löwer, V. N. Kondrashov, M. Basko, A. Kendl, J. Meyer-ter-Vehn, R. Sigel and A. Ng, Reflectivity and optical brightness of laser-induced shocks in silicon, Phys. Rev. Lett., 80, 4000 (1998).

[45] S. N. Luo, J. A. Akins, T. J. Ahrens and P. D. Asimow, Shock-compressed $\mathrm{MgSiO}_{3}$ glass, enstatite, olivine, and quartz: optical emission, temperatures, and melting, J. Geophys. Res., 109, B05205 (2004).

[46] M. A. Meyers, F. Gregori, B. K. Kad, M. S. Schneider, D. H. Kalntar, B. A. Remington, J. S. Wark, T. Boehly and G. Ravichandran, Plastic deformation in laser-induced shock compression of monocrystalline copper, In: M. D. Furnish, N. N. Thadhani and Y. Horie (Eds.), Shock Compression of Condensed Matter - 2001 (AIP Conf. Proc., CP620) (Am. Inst. Phys., Melville, 2002) pp. 619-622.

[47] T. de Resseguier, P. Berterretche, M. Hallouin and J. P. Petitet, Structural transformations in laser shock-loaded quartz, J. Appl. Phys., 94, 2133 (2003).

[48] S. N. Luo, O. Tschauner, P. D. Asimow and T. J. Ahrens, A new dense silica polymorph: A possible link between tetrahedrally and octahedrally coordinated silica, Am. Mineral., 89, 455 (2004).

[49] M. O'Keeffe, G. B. Adams and O. F. Sankey, Predicted new low energy forms of carbon, Phys. Rev. Lett., 68, 2325 (1992). 